\title{
Need for a standardized protocol for stress echocardiography in provoking subaortic and valvular gradient in various cardiac conditions
}

\author{
Pawel Petkow Dimitrow ${ }^{1 *}$, Carlos Cotrim ${ }^{2}$ and Tsung $O$ Cheng $^{3}$
}

\begin{abstract}
(Semi) supine exercise testing has an established role in the evaluation of patients with valvular heart disease and can help clinical decision making. Stress echocardiography has the advantages of its wide availability, low cost, and versatility for the assessment of disease severity. However, exercise-induced changes in valve hemodynamics, left ventricular outflow obstruction and pulmonary artery pressure depended on load variation. Changing position from supine to upright rapidly decreases load conditions for the ventricles. Therefore several cardiac centers have proposed exercise stress echocardiography in the upright position with gradient monitoring sometimes also in post-exercise recovery. Doppler measurement of subaortic gradient has been a very helpful and informative examination in several heart diseases (especially in hypertrophic cardiomyopathy, valve heart diseases, prosthesis dysfunction).
\end{abstract}

Keywords: Exercise, Echocardiography, Stress test, Upright, Hypertrophic cardiomyopathy, valve stenosis

\section{Introduction}

Stress echocardiography has been introduced many years ago as a valuable method in the detection of myocardial ischemia in patients with known or suspected coronary artery disease by assessing wall motion abnormalities [1]. At least in some centers two-dimensional stress echocardiography is performed in the standing position throughout the exercise stress test with image acquisition at peak exercise [2]. Standing position, both at rest and during exercise, is a normal and fundamental activity of daily life, but may precipitate an unexpected fall in cardiac patients predisposed to syncope, especially in patients with unsuspected aortic or sub-aortic obstruction. Stress echocardiography is not only useful for diagnosis of coronary artery disease but also for Doppler measurement of sub-aortic valvular pressure gradient.

The evaluation of sub-aortic obstruction only at rest might underestimate the full impact of the lesion and its clinical effects. In a pioneering study published in 1966, Mason, Braunwald and Ross [3] reported that cardiac symptoms in these patients were noted most commonly when they were in the erect position, and

\footnotetext{
* Correspondence: dimitrow@mp.pl

'2nd Department of Cardiology CMUJ, 31-501 Cracow, Poland

Full list of author information is available at the end of the article
}

these symptoms also tended to occur during or immediately after exertion.

\section{Exercise test protocol}

The exercise protocol is a complex issue. Currently there are 3 protocols used for stress echocardiography in provoking or exacerbating left ventricular outflow tract gradient (LVOTG) in patients with hypertrophic cardiomyopathy (HCM):

1. Fully-physiological: upright position during both exercise and recovery with continuous echocardiographic monitoring of LVOTG (recommended in our opinion)

2. Non-physiological: supine position at both stages with echocardiographic monitoring

3. Semi-physiological: treadmill exercise followed by echocardiographic recording at recovery in a supine position.

Supine exercise is technically less demanding but also less physiological than upright exercise. It should be underscored that orthostatic exercise reflects physical exercise during everyday activity and reduces the preload more than supine exercise. 
Table 1 Proposal of Echo-Doppler exercise echocardiography

\begin{tabular}{ll}
\hline Protocol & Comments, references \\
\hline 1. Period of 12-h fasting & {$[7]$}
\end{tabular}

before exercise

2. Physiological exercise test for LVOTG provocation

A/ Pre-exercise stage - echocardi ography first in supine position and then in upright position

B/ Position during

exercise - upright

C/ Exercise gradient

monitoring - continuous

D/ Moment of gradient measurement by Doppler echocardiography - peak exercise

E/ Mode of exercise - treadmill

F/ Termination of test symptom-limited;

In some patients rapid emergence or increase in LVOTG has been reported after mere standing prior to orthostatic stress testing; under such circumstances, exercise provocation is not only unnecessary but even contraindicated, because of the risk of syncope.

bicycle can be confidently used to acquire peak images, but peak $\mathrm{O}_{2}$ consumption is lower than that achieved by treadmill, which might lead to lower sensitivity. In addition, in contrast to treadmill, leg discomfort or lack of leg strength is a common reason for terminating prematurely the bicycling test.

alternatively, pre-specified heart rate/workload (or combination).

G/ Post-exercise recovery -

continuous monitoring of

LVOTG in upright position

Full protocol in treadmill 2A-G [8-13]

Recently, Lafitte et al. [4] clearly documented that the $2^{\text {nd }}$ approach could not be considered to be a pure evaluation of exercise dynamics and also postulated that $3^{\text {rd }}$ option is not adequate because dramatic pre-load variations were observed a few seconds after the end of exercise.

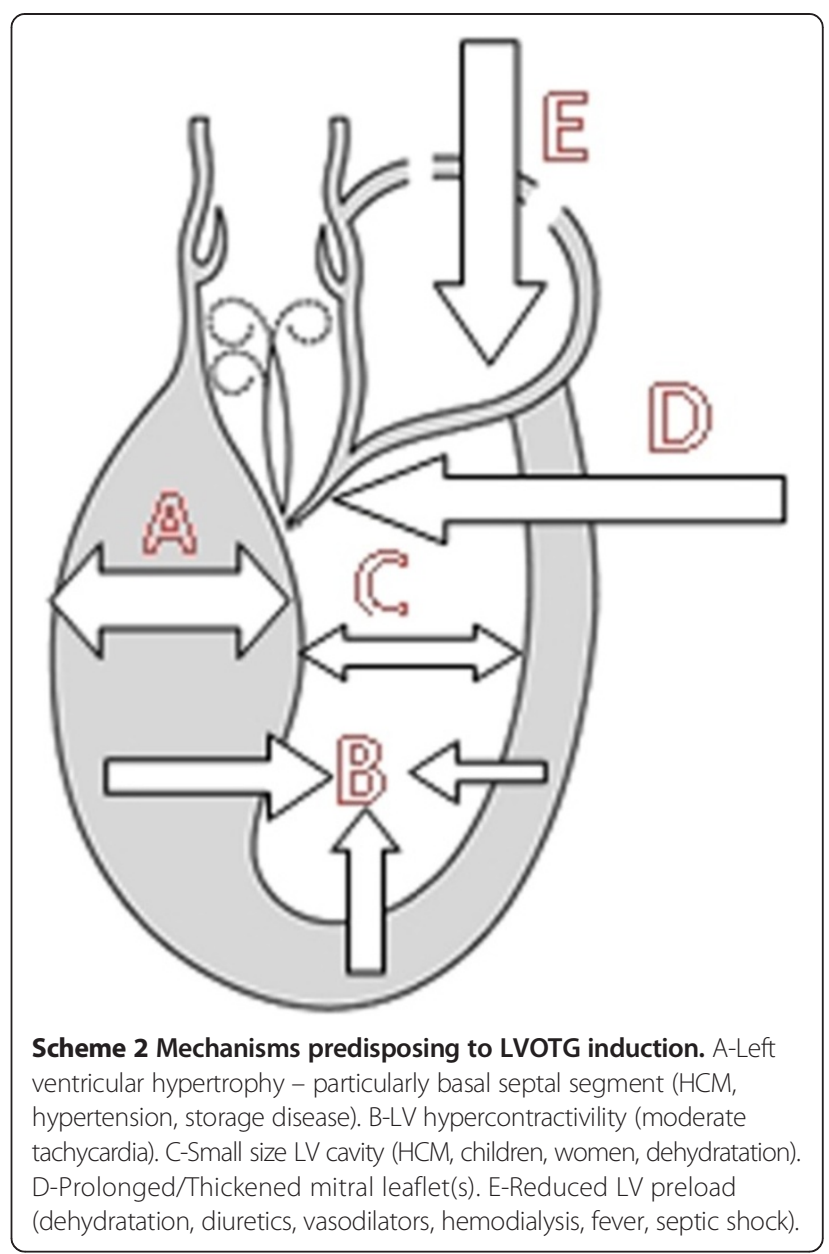

They [4] further stressed that, when the subject was upright at the termination of exercise, there was a large decrease in venous blood return to the heart, yielding a decreased left ventricular volume, a decreased wall stress, a continued sympathetic drive, and a hyperkinetic state like that observed during dobutamine-induced stress.

In the two most recent publications, Wittlieb-Weber et al. $[5,6]$ stated that, since upright positioning was

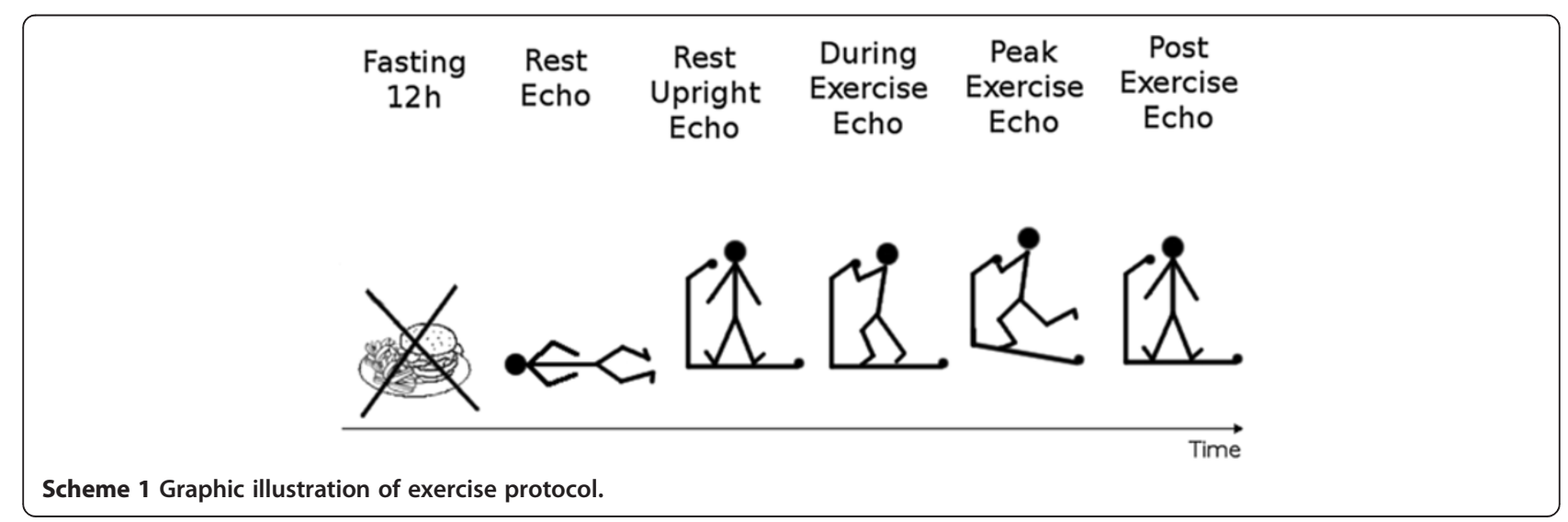


Table 2 Doppler echo exercise upright treadmill/bicycle test - examples of some centers and examined diseases/conditions

\begin{tabular}{ll}
\hline Country, town of center, type of exercise machine & Measured gradie \\
\hline 1. Portugal, Almada, treadmill & \\
- Hypertrophic cardiomyopathy [8,14], & LVOT \\
-Symptomatic athletes (Intra-ventricular obstruction & \\
induced by exercise in athletes with "positive screening" & LVOT \\
in medical evaluation for sports practice, [9] & \\
- Cardiac syndrome X [10] & LVOT \\
- Pulmonary artery hypertension [11] & Transtricuspid \\
- Mitral stenosis [12] & \\
-Aortic stenosis [13] & Tranmitral
\end{tabular}

2. Poland, Cracow, treadmill

- Hypertrophic cardiomyopathy $[15,16]$

3. USA, Philadelphia, treadmill

- "healthy" youth for cardiac evaluation due palpitations, syndrome WPW, short of breath, chest pain [5]

4. Sweden, Vasteras, bicycle

- Healthy athletes males [17]

5. USA, Spriengfield/ England, Liverpool, bicycle

- Healthy adolescent boys and girls [18]

6. England, London, bicycle

- Hypertrophic cardiomyopathy [19]

- Anderson-Fabry disease [20]

7. Spain, A Coruna, treadmill

- Hypertrophic cardiomyopathy [21]

8. Spain, Malaga, treadmill

- Effort angina [22]

9. Altavilla Vicentina, Italy, treadmill (comparison with semi physiological test protocol)

- Hypertrophic cardiomyopathy [23]

10. Germany, Munster, bicycle

- Hypertrophic cardiomyopathy [24]

11. Korea, Seoul, treadmill

-Aortic stenosis [25]

12. USA, Cleveland, treadmill

-Hypertrophic cardiomyopathy [26]

13. Canada, Quebec, bicycle

LVOT

LVOT

Clinical impact of exercise echo in UPRIGHT position

Stratification risk of sudden death in HCM because LVOTG is risk factor

Assessment of pharmacological and nonpharmacological methods reducing LVOTG

Verification of exercise-induced symptoms in relation to LVOTG

Monitoring treatment with beta blockers

Verification of exercise-induced symptoms in relation to LVOTG

Monitoring the use of beta blockers

Verification of exercise-increase of pulmonary hypertension (assessment of dyspnea)

Verification of exercise-induced symptoms in relation to trasmitral gradient

Verification of exercise-induced symptoms in relation to transaortic gradient

HCM see above

Hemodynamic verification of symptoms pathophysiology

LVOT

Transaortic

Transaortic

LVOT

LVOT

LVOT

LVOT

Transaortic

LVOT

Transprothesis- Aortic
Definition of normal value

Definition of normal value

HCM see above

Hemodynamic assessment the effect of myocardial hypertrophy induced by storage disease

HCM see above

Verification of exercise-induced symptoms Monitoring the effect of beta blockers

HCM see above

HCM see above

Verification of exercise-induced symptoms in relation to transaortic gradient

HCM see above

Assessment of prosthesis function and 'patients-prosthesis mismatch' phenomenon 
Table 2 Doppler echo exercise upright treadmill/bicycle test - examples of some centers and examined diseases/conditions (Continued)

- Patients with a bioprosthesis in the aortic valve position

[27], bicycle

- Comparison of stentless versus stented bioprostheses in aortic valvular position [28], bicycle

more physiologic, it seems logical that this would be the standard approach for LVOTG assessment. According to these authors, since increasingly more studies had been, and would be, published in evaluating the LVOTG by exercise stress echocardiography, standardization of this measurement and specific guidelines on stress echocardiography for this indication, which thus far had been lacking, should be stipulated.

\section{Methodology}

The specific preparation for exercise test for echocardiographic monitoring has been described in detail by Cotrim et al. [2]. Also, the echocardiographic technology in upright position has been precisely demonstrated in this publications. At the present moment, we would like to propose a standardized stress echocardiographic protocol for LVOTG provocation in HCM in accordance with several previous studies exploring not only HCM but also other cardiac conditions (Table 1, Scheme 1). Mechanisms predisposing to LVOT induction are summarized in Scheme 2. The Doppler-echocardiographic approach is from the apical view.

\section{Investigation centers/cardiac diseases}

We have performed systematic search across the publication database PubMed using combination of keywords: "echocardiography”, "exercise", gradient”. We came across (as of May 12-15, 2014) 468 adequate publications concerning body position during exercise and moments of Doppler measurements (minimal including criteria for analysis presented in Table 2 must fulfill at a minimum items B and D proposed in Table 1).

In transaortic valve level, normal values for healthy subjects during upright bicycle exercise in 24 adult healthy male endurance athletes from rest to peak exercise (at a heart rate of $160 \mathrm{bpm}$ ) are as follows:, the maximum aortic flow velocity almost doubled $(1,14$ vs $2,20 \mathrm{~m} / \mathrm{s})$ and the maximum transmitral flow velocity more than doubled $(0,62$ vs $1,43 \mathrm{~m} / \mathrm{s})$ [17]. The transaortic velocities with the similar increases were achieved in untrained adolescent boys (1,36 vs 2,08 m/s) and girls (1,08 vs $1,96 \mathrm{~m} / \mathrm{s})$ [18]. Maximal upright exercise transaortic gradient should be peaked below $20 \mathrm{mmHg}$ in bicycle exercise.

We would like to emphasize that (sub)valvular gradient measurement during and after exercise may be diagnostically useful also in other diseases/conditions than HCM. Therefore, a standardized exercise protocol is of paramount importance for universal application in the practice of cardiology (Table 2), (Figures 1, 2, 3, 4 and 5), (Additional file 1, Additional file 2, and Additional file 3). In Table 2, we have included studies with at least Items B and D from Table 1. A significant number of studies on

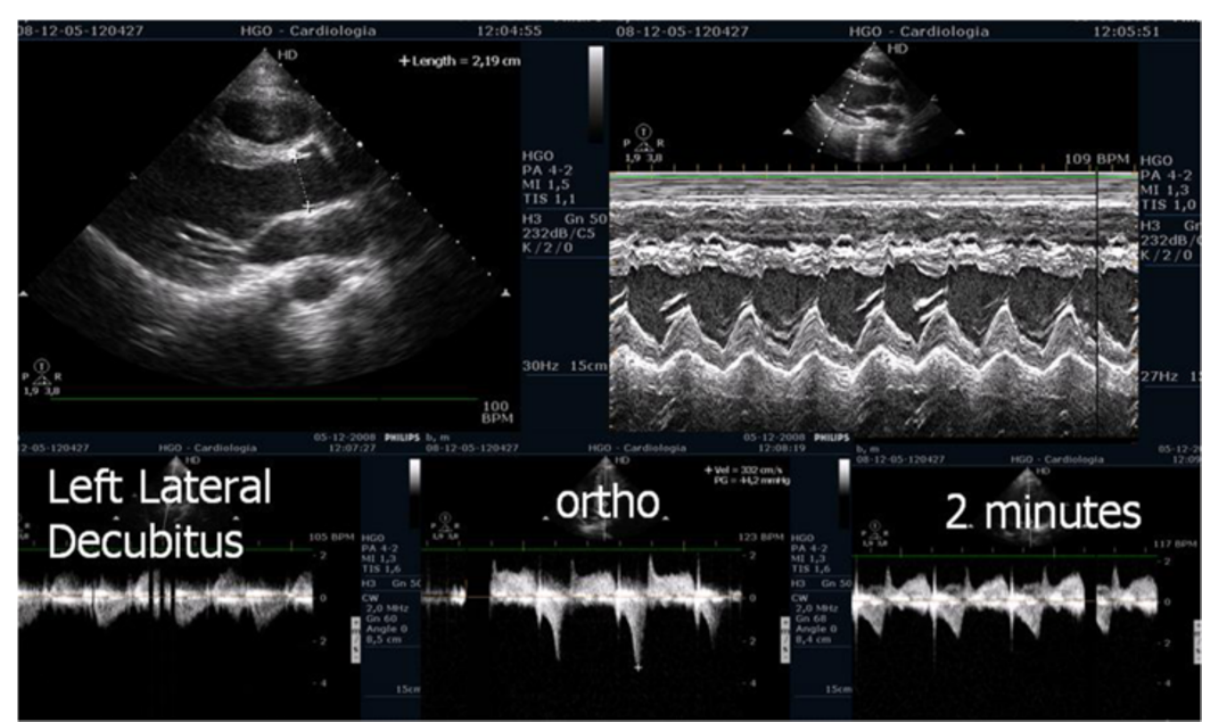

Figure 1 Echocardiogram before exercise with symptomatic athlete in left lateral decubitus position and in orthostatic position before and at beginning of exercise. 


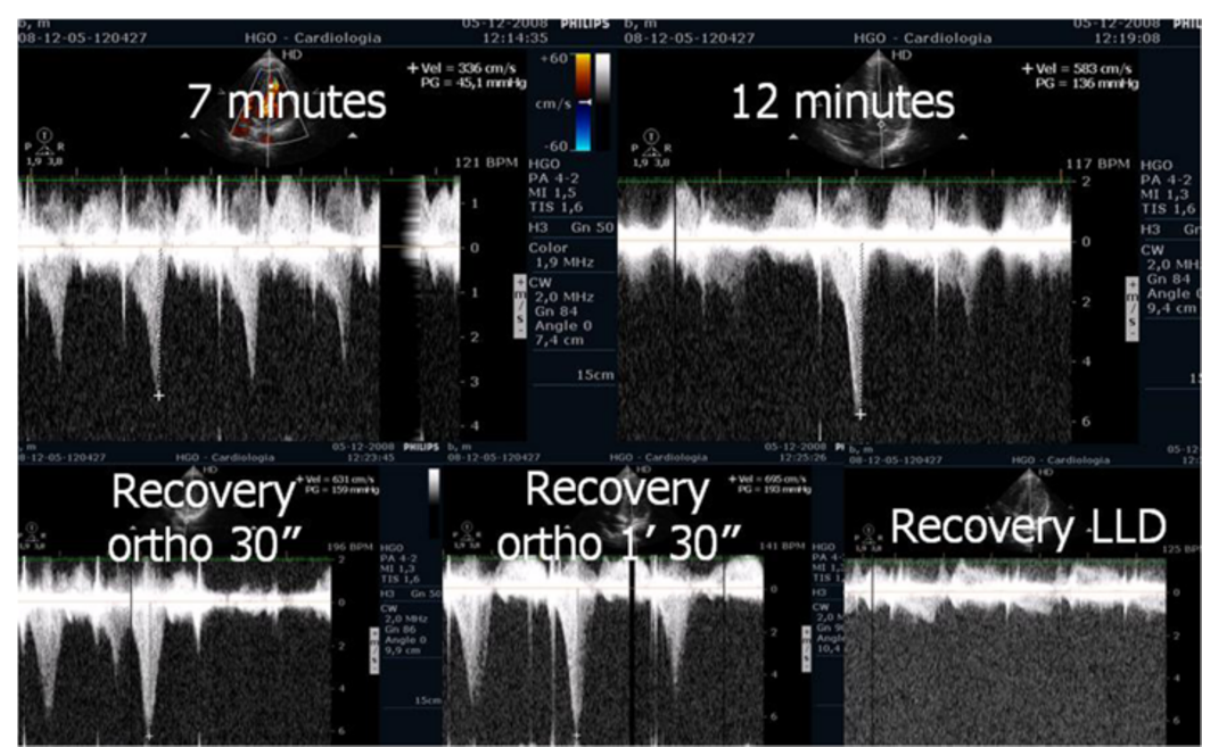

Figure 2 Intraventricular gradient in the various phases of exercise in same symptomatic athlete.

upright bicycle exercise was used; thus our analysis is heterogeneous. Items 1 and 2 in Table 2 described our own experiences.

\section{Limitation of method and learning curves}

Elderly patients may not exercise well in supine positions [29]. On the other hand, treadmill exercise may predispose to syncope [30]; however, particularly in patients with a history of syncope, we should assess hemodynamic changes during treadmill exercise as potential risk factors.
An additional advantage of treadmill exercise is the fact that patients usually can achieve higher workload.

The main limitation of exercise stress echocardiography is the presence of a poor acoustic window in some patients $[31,32]$. Furthermore, only apical view is imaginable. However, in Doppler-gradient examination feasibility is high and success rate may be achieved in more than $90 \%$ of the cases. The subvalvular LVOT gradient is easier to measure than the transvalvular gradient in aortic valvular stenosis [33]. In some clinical situation (hemodialysis

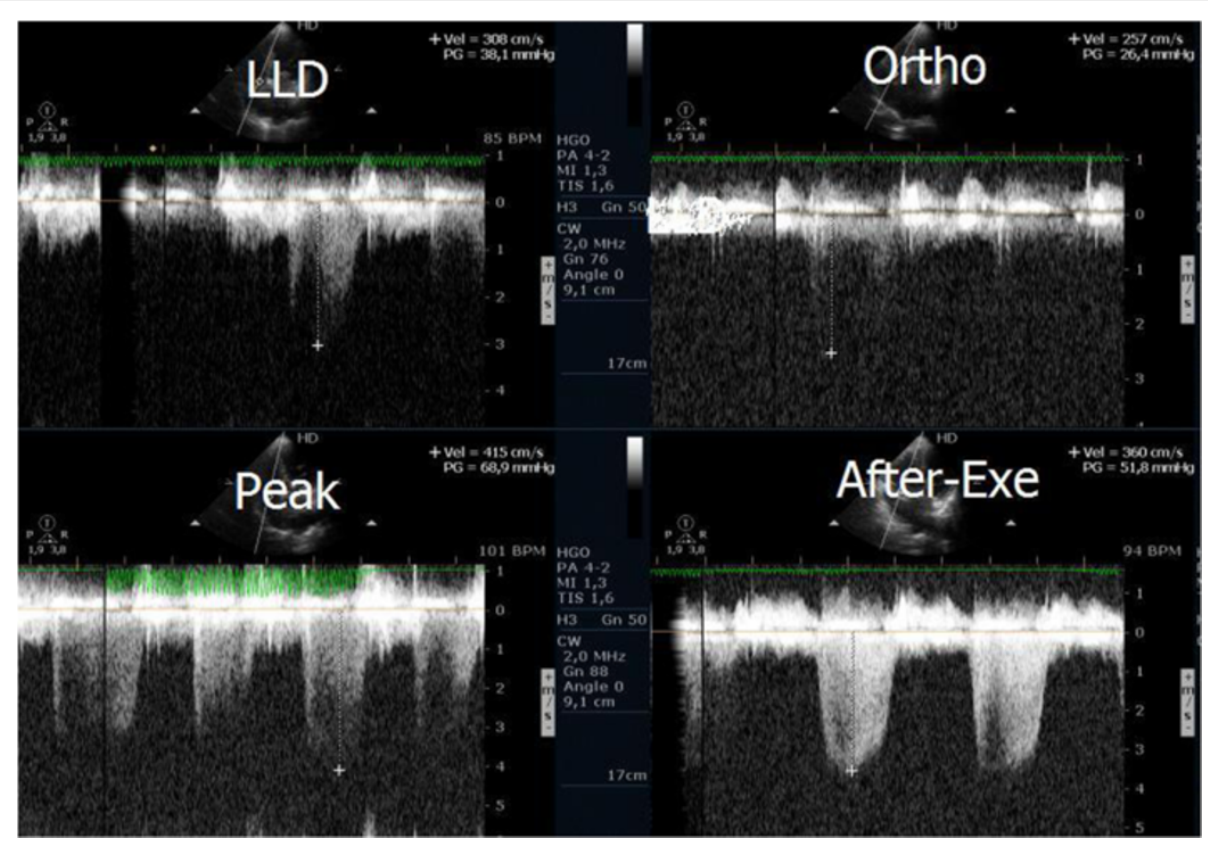

Figure 3 Right ventrícle /Right atrium gradient at different stages of the study in a patient with mitral stenosis. 


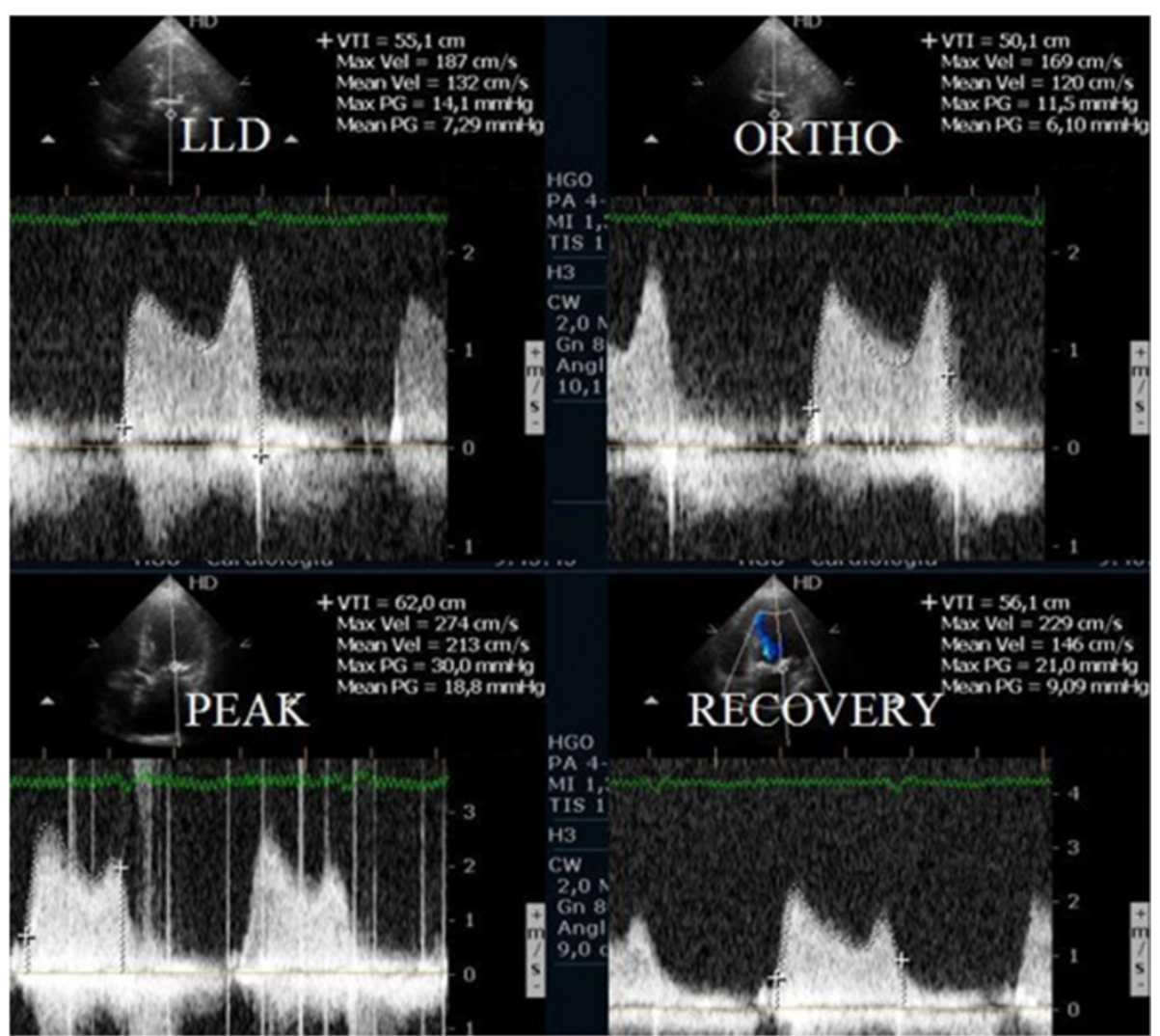

Figure 4 Left atrium/ left ventricle mean gradient, evaluated with CW Doppler, at different stages of the study in one patient with mitral stenosis.

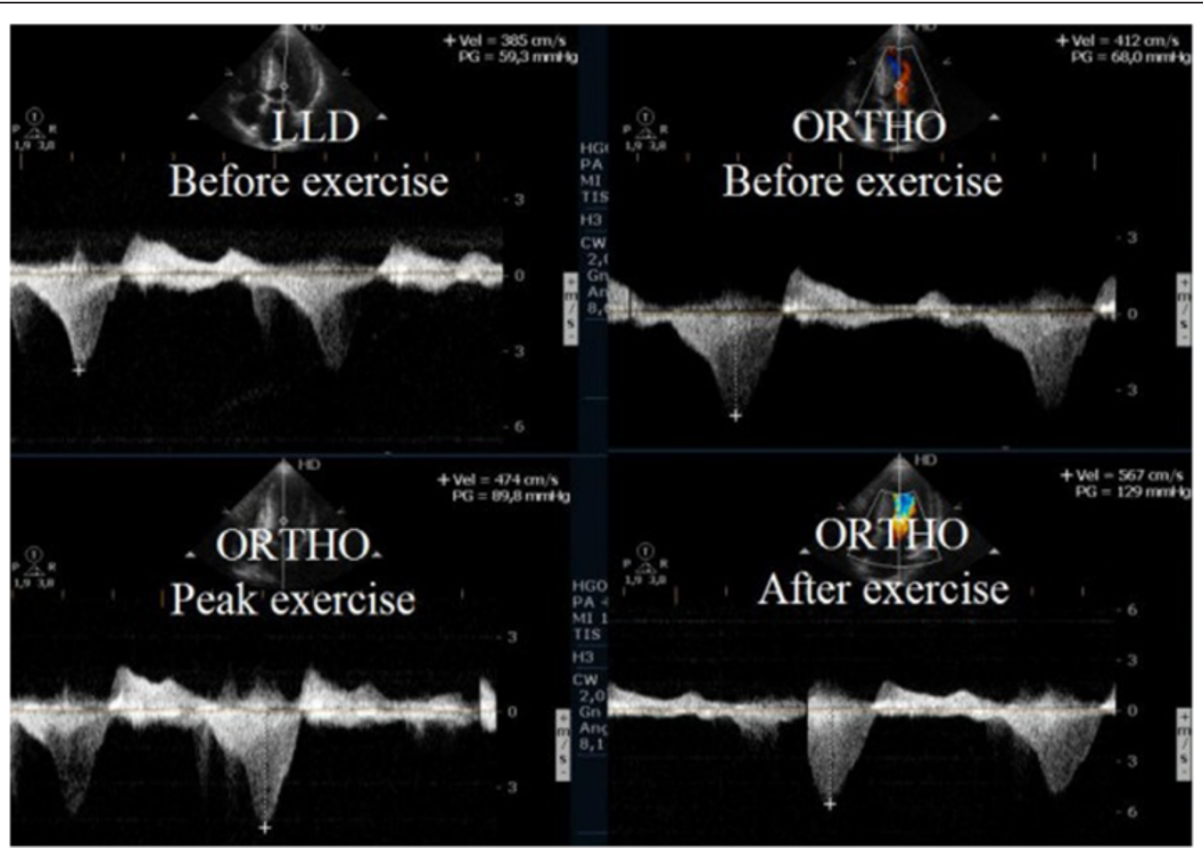

Figure $\mathbf{5}$ Intra-ventricular gradient present in all phases of the study in a HCM patient with increase also after exercise in orthostatic position. 
room) only passive (non-exercise) orthostatic test may be applicable [34].

Imaging acquisition during exercise echocardiography is more difficult than during pharmacological stress, due to the greater increase in both heart and respiratory rates with exercise. Pharmacological stress echocardiography requires less skills than exercise stress echocardiography. On the other hand, most physicians and fellows in training acquire the necessary expertise to perform peak exercise studies on a treadmill with confidence after 100 cases.

\section{Advantages of method}

The low cost, safety [35], diagnostic accuracy, possibility of evaluation of functional capacity and lack of radiation exposure should make exercise stress echocardiography an attractive procedure for patients with hypertrophic subvalvular or valvular aortic stenosis. The possibility of evaluation of Doppler data during and after exercise in orthostatic position in patients with LVOT obstruction provides very practical and useful information. Despite its limitations we believe that this test when standardized is suitable for use in every institution around the world.

\section{Conclusions}

Doppler gradient measurement during, and symptomatic responses to, exercise provides the clinicians with important diagnostic and prognostic information that can contribute to subsequent clinical decisions and management $[30,36]$.

Standing should be recommended as a physiologic provocative maneuver. In some patients, standing may guide therapy; in others, the exercise gradient measured in standing position provides a correct appreciation of the range of physiologically experienced gradients during normal daily upright activity.

Doppler echocardiography during and after upright exercise increases both the quality and quantity of information obtained in not only HCM but also many other clinical conditions. The preference of upright position is confirmed by positive experiences in many echocardiographic laboratories around the world.

\section{Additional files}

Additional file 1: Apical four-chamber view obtained in apical window before exercise in one patient with "non obstructive" HCM containing two dimensional data (with SAM).

Additional file 2: Apical four-chamber view obtained in apical window at peak exercise in the same patient with SAM causing severe obstruction.

Additional file 3: Apical four-chamber view obtained in apical window after exercise in the same patient with SAM causing severe obstruction.

\section{Abbreviations}

LVOTG: Left ventricular outflow tract gradient; HCM: Hypertrophic cardiomyopathy.
Competing interests

The authors declare that they have no competing interests.

\section{Authors' contributions}

All Authors have been involved in drafting the manuscript or revising it critically for important intellectual content and have given final approval of the version to be published.

\section{Author details}

2nd Department of Cardiology CMUJ, 31-501 Cracow, Poland. ${ }^{2}$ Circulation Department, Hospital da Cruz Vermelha Portuguesa, Lisboa, Portugal. ${ }^{3}$ Department of Medicine, The George Washington University Medical Center, 2150 Pennsylvania Avenue, NW, Washington, DC 20037, USA.

Received: 26 April 2014 Accepted: 30 June 2014

Published: 14 July 2014

\section{References}

1. Peteiro J, Fabregas R, Montserrat L, Martinez D, Castro-Beiras A: Comparison of treadmill exercise echocardiography before and after exercise in the evaluation of patients with known or suspected coronary artery disease. J Am Soc Echocardiogr 1999, 12:1073-1079.

2. Cotrim C, João I, Fazendas P, Almeida AR, Lopes L, Stuart B, Cruz I, Caldeira D, Loureiro MJ, Morgado G, Pereira H: Clinical applications of exercise stress echocardiography in the treadmill with upright evaluation during and after exercise. Cardiovasc Ultrasound 2013, 11:26.

3. Mason DT, Braunwald E, Ross J: Effects of changes in body position on the severity of obstruction to left ventricular outflow in idiopathic subaortic stenosis. Circulation 1966, 33:374-382.

4. Lafitte S, Reant P, Touche C, Pillois X, Dijos M, Arsac F, Peyrou J, Montaudon M, Ritter $P$, Roudaut $R$, Demaria A: Paradoxical response to exercise in asymptomatic hypertrophic cardiomyopathy: a new description of outflow tract obstruction dynamics. J Am Coll Cardiol 2013, 62:842-850.

5. Wittlieb-Weber CA, Cohen MS, McBride MG, Paridon SM, Stephens P Jr: Elevated left ventricular outflow tract velocities on exercise stress echocardiography may be a normal physiologic response in healthy youth. J Am Soc Echocardiogr 2013, 26:1372-1378.

6. Wittlieb-Weber CA, Cohen MS, McBride MG, Paridon SM, Stephens P Jr: Authors' reply. J Am Soc Echocardiogr 2014, 27:341-342.

7. Feiner E, Arabadjian M, Winson G, Kim B, Chaudhry F, Sherrid M: Postprandial upright exercise echocardiography in hypertrophic cardiomyopathy. J Am Coll Cardiol 2013, 61:2487-2493.

8. Cotrim C, Loureiro MJ, Simoes O, Miranda R, Cordeiro P, lalá M, Matias C, João I, Carrageta M: Evaluation of hypertrophic obstructive cardiomyopathy by exercise stress echocardiography: new methodology. Rev Port Cardiol 2005, 24:1319-1327.

9. Cotrim C, Miranda R, Loureiro MJ, Almeida S, Lopes L, Almeida AR, Fernandes R, Simões O, Cordeiro A, Cordeiro P, Carrageta M: Echocardiography during treadmill exercise testing for evaluation of pulmonary artery systolic pressure: advantage of the method. Rev Port Cardiol 2008, 27:453-461.

10. Almeida AR, Cotrim C, Miranda R, Almeida S, Lopes L, Loureiro MJ, Simões O, Cordeiro P, Fazendas P, João I, Carrageta M: Echocardiography during treadmill exercise testing in a patient with mitral stenosis. Rev Port Cardiol 2009, 28:195-199.

11. Cotrim C, Almeida AG, Carrageta M: Exercise-induced intra-ventricular gradients as a frequent potential cause of myocardial ischemia in cardiac syndrome X patients. Cardiovasc Ultrasound 2008, 6:3.

12. Cotrim C, Loureiro MJ, Miranda R, Almeida AG, Cotrim H, Andrade JP, Picano E, Carrageta M: Efficacy of beta-blocker therapy in symptomatic athletes with exercise-induced intra-ventricular gradients. Cardiovasc Ultrasound 2010, 8:38.

13. Piçarra BC, Miranda R, Cotrim C, Almeida AR, Lopes L, João I, Pereira H: Dyspnea in aortic stenosis: appearences can be deceptive. Rev Port Cardiol 2012, 31:27-30

14. Miranda R, Cotrim C, Cardim N, Almeida S, Lopes L, Loureiro MJ, Simões O, Cordeiro P, Fazendas P, João I, Carrageta M: Evaluation of left ventricular outflow tract gradient during treadmill exercise and in recovery period in orthostatic position, in patients with hypertrophic cardiomyopathy. Cardiovasc Ultrasound 2008, 6:19. 
15. Dimitrow PP, Bober M, Michałowska J, Sorysz D: Left ventricular outflow tract gradient provoked by upright position or exercise in treated patients with hypertrophic cardiomyopathy without obstruction at rest. Echocardiography 2009, 26:513-520.

16. Dimitrow PP, Cheng TO: Standing position alone or in combination with exercise as a stress test to provoke left ventricular outflow tract gradient in hypertrophic cardiomyopathy and other conditions. Int J Cardiol 2010, 143:219-222.

17. Sundstedt M, Hedberg P, Jonason T, Ringqvist I, Henriksen E: Doppler assessments of left ventricular filling and ejection during upright exercise in endurance athletes. Clin Physiol Funct Imaging 2007, 27:36-41.

18. Rowland T, Unnithan V, Garrard M, Roche D, Holloway K, Sandoval J, Marwood S: Sex influence on myocardial function with exercise in adolescents. Am J Hum Biol 2010, 22:680-682.

19. Shah JS, Tome Esteban MT, Thaman R, Sharma R, Mist B, Pantazis A, Ward D, Kohli SK, Page SP, Demetrescu C, Sevdalis E, Keren A, Pellerin D, McKenna WJ, Elliott PM: Prevalence of exercise induced left ventricular outflow tract obstruction in symptomatic patients with non-obstructive hypertrophic cardiomyopathy. Heart 2008, 94:1288-1294.

20. Calcagnino M, O'Mahony C, Coats C, Cardona M, Garcia A, Janagarajan K, Mehta A, Hughes D, Murphy E, Lachmann R, Elliott PM: Exercise-induced left ventricular outflow tract obstruction in symptomatic patients with Anderson-Fabry disease. J Am Coll Cardiol 2011, 58:88-89.

21. Peteiro J, Bouzas-Mosquera A, Fernandez X, Monserrat L, Pazos P, EstevezLoureir $R$, Castro-Beiras A: Prognostic value of exercise echocardiography in patients with hypertrophic cardiomyopathy. J Am Soc Echocardiogr 2012, 25:182-189.

22. Cabrera-Bueno F, Pinilla JMG, Doblas JJG, Montiel-Trujillo A, Rodríguez-Bailón I, de Teresa-Galván E: Beta-blocker therapy for dynamic left ventricular outflow tract obstruction induced by exercise. Int J Cardiol 2007, 117:222-226.

23. Nistri S, Olivotto I, Maron MS, Grifoni C, Baldini K, Baldi M, Sgalambro A, Cecchi F, Maron BJ: Timing and significance of exercise-induced left ventricular outflow tract pressure gradients in hypertrophic cardiomyopathy. Am J Cardiol 2010, 106:1301-1306.

24. Schwammenthal E, Schwartzkopff B, Block M, Johns J, Lösse B, Engberding R, Borggrefe M, Breithardt G: Döppler echocardiographic assessment of the pressure gradient during bicycle ergometry in hypertrophic cardiomyopathy. Am J Cardiol 1992, 69:1623-1628.

25. Cho EJ, Park SJ, Song JE, Kim SH, Lee YJ, Gak JH, Chang SA, Lee SC, Park SW: What is the real practice of exercise echocardiographic testing in asymptomatic patients with severe aortic stenosis? Chin Med J (Engl) 2013, 126:4649-4654.

26. Desai MY, Bhonsale A, Patel P, Naji P, Smedira NG, Thamilarasan M, Lytle BW, Lever HM: Exercise echocardiography in asymptomatic HCM: exercise capacity, and not LV outflow tract gradient predicts long-term outcomes. JACC Cardiovasc Imaging 2014, 7:26-36.

27. Pibarot $P$, Dumesnil JG, Jobin J, Lemieux M, Honos G, Durand LG: Usefulness of the indexed effective orifice area at rest in predicting an increase in gradient during maximum exercise in patients with a bioprosthesis in the aortic valve position. Am J Cardiol 1999, 83:542-546.

28. Pibarot $P$, Dumesnil JG, Jobin J, Cartier P, Honos G, Durand LG: Hemodynamic and physical performance during maximal exercise in patients with an aortic bioprosthetic valve: comparison of stentless versus stented bioprostheses. J Am Coll Cardiol 1999, 34:1609-1617.

29. Sicari R, Nihoyannopoulos P, Evangelista A, Kasprzak J, Lancellotti P, Poldermans D, Voigt JU, Zamorano JL: European Association of Echocardiography. Stress echocardiography expert consensus statement: European Association of Echocardiography (EAE) (a registered branch of the ESC). Eur J Echocardiogr 2008, 9:415-437.

30. Picano E, Pibarot $P$, Lancellotti $P$, Monin JL, Bonow RE: The emerging role of exercise testing and stress echocardiography in valvular heart disease. J Am Coll Cardiol 2009, 54:2251-2260.

31. Peteiro J, Bouzas-Mosquera A: Peak treadmill exercise echocardiography: not feasible? Eur Heart J 2009, 30:740

32. Peteiro J, Bouzas-Mosqueras A: Exercise echocardiography. World J Cardiol 2010, 2:223-232

33. Dimitrow PP, Sorysz D: Orthostatic stress echocardiography as a useful test to measure variability of transvalvular pressure gradients in aortic stenosis. Cardiovasc Ultrasound 2013, 11:15.
34. Dimitrow PP, Michałowska J, Sorysz D: The effect of hemodialysis on left ventricular outflow tract gradient. Echocardiography 2010, 27:603-607.

35. Fennich N, Ellouali A, Abdelali S, Chaara A, Berrada A, Imane C: Stress echocardiography: safety and tolerability. Cardiovasc Ultrasound 2013, 11:30.

36. Picano E, Pellikka PA: Stress echo applications beyond coronary artery disease. Eur Heart J 2014, 35:1033-1040.

doi:10.1186/1476-7120-12-26

Cite this article as: Petkow Dimitrow et al:: Need for a standardized protocol for stress echocardiography in provoking subaortic and valvular gradient in various cardiac conditions. Cardiovascular Ultrasound $201412: 26$

\section{Submit your next manuscript to BioMed Central and take full advantage of:}

- Convenient online submission

- Thorough peer review

- No space constraints or color figure charges

- Immediate publication on acceptance

- Inclusion in PubMed, CAS, Scopus and Google Scholar

- Research which is freely available for redistribution 